\title{
Rotating Black Hole, Twistor-String and Spinning Particle
}

\author{
Alexander Burinskii \\ NSI Russian Academy of Sciences, \\ B.Tulskaya 52, Moscow 115191, RUSSIA *
}

August 16, 2018

\begin{abstract}
We discuss basic features of the model of spinning particle based on the Kerr solution. It contains a very nontrivial real stringy structure consisting of the Kerr circular string and an axial stringy system.

We consider also the complex and twistorial structures of the Kerr geometry and show that there is a complex twistor-string built of the complex $\mathrm{N}=2$ chiral string with a twistorial $(x, \theta)$ structure. By imbedding into the real Minkowski $\mathbf{M}^{4}$, the $\mathrm{N}=2$ supersymmetry is partially broken and string acquires the open ends. Orientifolding this string, we identify the chiral and antichiral structures. Target space of this string is equivalent to the Witten's 'diagonal' of the $\mathbf{C P}^{\mathbf{3}} \times \mathbf{C P}^{* 3}$.
\end{abstract}

\section{Introduction}

In the recent paper [1] Witten suggested a new fundamental object, twistorstring, incorporating twistors in superstring theory. It was conjectured that the twistor-string controls the scattering processes and is responsible for analytic properties of the scattering amplitudes, and therefore, this string may

*Talk given at the Conference 'Symmetries and Spin'(SPIN-Praha-2004) July 2004. 
be an element of the structure of fundamental particles. This Witten paper and the subsequent papers have paid extraordinary attention which has been heated by the progress in computation of some scattering amplitudes on the base of twistor inspired methods $[2,3]$. In particular, the striking simplifications of the maximally helicity violating (MHV) amplitudes were achieved by the calculations performed on the base of the spinor helicity formalism (for review see for example $[1,4]$ ).

In this paper we consider the model of spinning particle [5] which is based on the Kerr rotating black hole solution. The Kerr geometry has a remarkable twistorial structure, and the Kerr's spinning particle has also a very non-trivial stringy structure. The aim of this paper is to describe the complex, twistorial and stringy structures of the Kerr spinning particle and to argue that the new Witten's 'twistor-string' is related to the structure of the Kerr spinning particle, and therefore, the fusion of the twistor and string theories has a natural extension to the structure of the rotating black holes.

In Sec. 2 we give a very brief introduction to twistors, in.Sec.3 we treat briefly the structure of the Kerr spinning particle and its basic properties. In sec. 4 the twistorial structure of the Kerr geometry is discussed. In Sec.5 we consider the complex Kerr geometry and show that it contains a version of the complex twistor-string which has some properties of the well known $\mathrm{N}=2$ string [6] and also the chiral $(x, \theta)$ twistor-string $[7,8,9,10]$. Being imbedded into $\mathbf{M}^{4}$, this string turns out to be open with a partially broken $\mathrm{N}=2$ supersymmetry. The world-sheet of this string is formed by orientifolding the chiral and antichiral structures, and target space turns out to be equivalent to Witten's 'diagonal' of the $\mathbf{C P}^{\mathbf{3}} \times \mathbf{C P}^{* \mathbf{3}}$.

\section{Twistors}

Twistor may be considered as a world-line of the lightlike particle, which geometrically is a null line in Minkowski spacetime $\mathbf{M}^{\mathbf{4}}$ [11]. Momentum of the lightlike particle is described by the null vector $p^{\mu}$ satisfying the condition $p^{\mu} p_{\mu}=0$ and may be represented in the spinor form

$$
p^{\mu}=\psi \sigma^{\mu} \bar{\psi}
$$

where $\sigma^{\mu}$ are the Pauli matrices. A null line going via the coordinate origin may be described as $x^{\mu}(t)=p^{\mu} t$. 
We will also consider the complexified Minkowski space $\mathbf{C M}^{4}$. In this case $p^{\mu}=\psi \sigma^{\mu} \tilde{\psi}$, where $\tilde{\psi}$ and $\psi$ are independent spinors. Varying $\tilde{\psi}$ at fixed $\psi$ one obtains the (say "left" ) complex planes. Similar, varying $\psi$ at fixed $\tilde{\psi}$ one obtains another family of the ("right") complex planes. They are totally null planes in the sense that any vector lying in them is null. Therefore, from the complex point of view twistors are complex null planes. Intersection of the complex conjugated left and right planes yields the real twistor.

The main difference between twistors and spinors is that twistors describe the null lines which has a definite position with respect to the origin of coordinates (or an observer). Correspondingly, the extra parameters are necessary to fix the position of line. If a point $x_{0}^{\mu}$ is fixed on the line, $x^{\mu}(t)=$ $x_{0}^{\mu}+p^{\mu} t$, it may be described by two spinors: $\psi^{\alpha}$ and $\omega_{\dot{\alpha}}=x_{0}^{\nu} \sigma_{\nu \alpha \dot{\alpha}}$. In the flat spacetime these parameters are independent of the position $x_{0}^{\nu}$ on the null line, and the four spinor parameters

$$
Z^{a}=\left\{\psi^{\alpha}, \omega_{\dot{\alpha}}\right\}
$$

give the basic representation of twistor. However, the initial set

$$
\left\{x_{0}^{\mu}, \psi^{\alpha}\right\}
$$

may also be considered as an equivalent representation which is used in the spinor helicity formalism [4]. Finally, since spinor $\psi^{\alpha}$ gives the homogenous coordinates of the null ray, one can use the equivalent projective spinor coordinate $Y=\psi_{2} / \psi_{1} \in \mathbf{C P}^{\mathbf{1}}$, and represent twistor in the form

$$
\left\{x_{0}^{\mu}, Y\right\}
$$

or via tree projective twistor coordinates

$$
\left\{Y=Z^{2} / Z^{1}, \quad \lambda_{1}=Z^{3} / Z^{1}, \quad \lambda_{2}=Z^{4} / Z^{1}\right\} \in \mathbf{C P}^{3} .
$$

The both forms (4) and (5) are used in the Kerr-Schild formalism [12] and in the description of twistor-string [1].

\section{The Kerr spinning particle: black hole which is neither 'black' nor 'hole'}

The joke in the title of this section belongs to P. Townsend. It has direct relation to the Kerr spinning particle. 
The Kerr geometry has found application in a very wide range of physical systems: from the rotating black holes and galactic nucleus to fundamental solutions of the low energy string theory. It must not be wonder that it has the relations to spinning particles too. Indeed, the Kerr-Newman solution has anomalous, $g=2$, gyromagnetic ratio of the Dirac electron. In the units $\hbar=c=G=1$, the parameters of the Kerr solution adapted to electron are $e^{2} \sim 1 / 137, \quad m \sim 10^{-22}, \quad a \sim 10^{22}, \quad m a=1 / 2$.

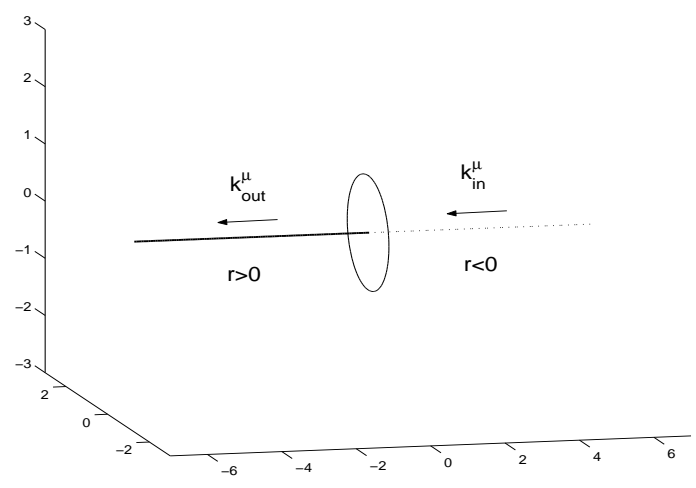

Figure 1: The Kerr singular ring and a semistring (or semitwistor) emerging from the 'negative' sheet of the Kerr space.

One sees that $a>>m$, and the Kerr black-hole horizons disappear, revealing the naked singular ring of the Compton radius $a \sim \hbar / m$. Metric is almost flat and gravitational field is concentrated in a very narrow vicinity of the singular ring.

The Kerr singular ring is the branch line of the Kerr space on two sheets, 'positive' $(r>0)$ and 'negative' $(r<0)$, where $r$ is radial coordinate of the oblate spheroidal coordinate system. The fields change directions and signs on the negative sheet. The negative sheet acquires an interpretation of the sheet of advanced fields which may be related to the vacuum zero point field $[13,14,15]$. The particles, strings or twistors disappear in (emerge from) a mirror world passing through the Kerr ring (see Fig. 1).

In the old paper [16] a model of the spinning Wheeler's geon was suggested, in which a quantum electromagnetic excitation - traveling waves along the Kerr ring generate the spin and mass of the geon. It was recognized soon [17] that the Kerr ring is a closed chiral string. 
The treatment of singular lines as strings is very natural. Among the well known examples are the fundamental strings of the low energy string theory $[18,19]$ and the strings interacting with Higgs field, in particular, the Nielsen-Olesen vortex line in a superconducting media and the Witten chiral superconducting cosmic string, where one of two Higgs fields is concentrated on the singular line [20]. It was shown in [21] that the field around the Kerr ring is similar to the field around the fundamental heterotic string, and finally, in [13] that the Kerr ring is a D-string with an orientifold world-sheet.

Recently, we have shown $[5,15]$ that the circular Kerr string is not unique in the Kerr spinning particle, and there is a second, 'axial' stringy system. It was shown that the wave excitations of the Kerr ring lead unavoidable to the appearance of the extra axial singular lines which are chiral strings similar to the Witten cosmic strings. Therefore, the Kerr spinning particle acquires a stringy skeleton forming by the Kerr circular string and by the axial stringy system consisting of two semi-infinite strings of opposite chiralities, see Fig. 2 .

The axial semistrings are not new objects in the Kerr geometry. In fact, the NUT-parameter of the Kerr-NUT solution led to the appearance of a semistring carrying the magnetic flow. Moreover, the general class of the Kerr-Schild solutions [12] contains a free holomorphic function $\psi(Y)$ which defines a series of the exact stationary solutions of the Einstein-Maxwell system containing singular semistrings.

In the simplest cases $\psi(Y) \sim Y^{n}$, where $n$ is integer ${ }^{1}$, and $Y$ has the form

$$
Y=e^{i \phi} \tan \frac{\theta}{2}
$$

in the Kerr oblate spheroidal coordinates. By $n<0$ solutions are singular at $\theta=0$, along the positive semiaxis $z^{+}$, and by $n>0$ solutions are singular at $\theta=\pi$, which corresponds to negative semiaxis $z^{-}$. The case $n=0$ corresponds to the charged Kerr-Newman field, and the axial singularity is absent. Up to our knowledge these solutions never were analyzed. In the case of spinning particle we have solutions with similar topological structure of singularities, however the function $\psi$ acquires an extra dependence on the retarded-time parameter $\tau$,

\footnotetext{
${ }^{1}$ Half-integer index $n$ can be considered in supergravity and also by twisting of spinstructure, see note in the Conclusion.
} 
$\psi \sim Y^{n} e^{i \omega \tau}$

As a result there appear traveling waves along the Kerr circular string and the chiral traveling waves along the axial semistrings[15, 5].

It was shown that the chiral excitations of the axial stringy system may be described by the Dirac equation. In the Weyl basis the Dirac current is represented as a sum of two lightlike components of opposite chiralities

$J_{\mu}=e\left(\bar{\Psi} \gamma_{\mu} \Psi\right)=e\left(\chi^{+} \sigma_{\mu} \chi+\phi^{+} \bar{\sigma}^{\mu} \phi\right)$.

The four-component spinor $\Psi=\left(\begin{array}{c}\phi_{\alpha} \\ \chi^{\dot{\alpha}}\end{array}\right)$, satisfies the massive Dirac equation which describes an interplay of two traveling waves of opposite chiralities - the lightlike fermions trapped by two axial singular semistrings. De Broglie periodicity appears as a result of the beating of these waves.

This axial stringy system plays an important role in the formation of the Kerr's twistor-string. We will show that two axial semistrings play the role of two D1-branes which are boundaries of the complex twistor-string.

It reproduces a very specific model of quarks: the fermionic currents of opposite chiralities adjoined to the ends of the twistor-string.

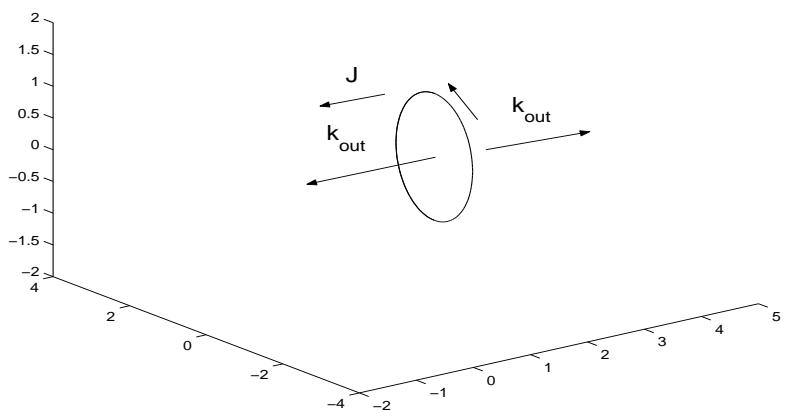

Figure 2: Stringy skeleton of the spinning particle. Circular string and two semi-infinite D-strings of opposite chiralities.

We summarize bellow the basic features and properties of the Kerr spinning particle:

- Anomalous gyromagnetic ratio $g=2$ of the Dirac electron, 
- The mass $m$, spin $J$ and charge $e$ are the only free parameters,

- Model is semiclassical - the quantum constant is used only to quantize energy of excitations,

- Particle has the Compton size $a=\hbar / m$, and the Compton region is stringy structured,

- Nontrivial real stringy structure: topological coupling of the axial and circular strings,

- Mass and spin have the origin from the circular traveling waves, electromagnetic excitations of the Kerr singular ring (the Wheeler's 'geon'),

- Axial stringy system is carrier of de Broglie waves which are described by the Dirac equation,

- Axial stringy system is responsible for the scattering at high energies,

- Remarkable complex and twistorial structures, twistor-string.

The following properties may also have a relation to the foundations of quantum theory ${ }^{2}$ :

- Wave function acquires a physical carrier - the axial stringy system,

- Axial string controls the motion of particle due to topological coupling of the axial and circular singular strings, so the model reproduces the features of the old wave-pilot conjecture by de Broglie.

- The quantum property - absence of radiation by oscillations - is exhibited here at the classical level, since the loss of energy by stringy excitations is compensated by the ingoing radiation from the 'negative' sheet of the Kerr spacetime.

\footnotetext{
${ }^{2}$ They were discussed in previous papers, see $[5,15]$ and reference therein
} 


\section{Kerr geometry in the Kerr-Schild formal- ism}

\subsection{Real Kerr geometry and twistors}

In the Kerr-Schild approach [12], the Kerr-Schild ansatz for metric is used

$$
g_{\mu \nu}=\eta_{\mu \nu}+2 h k_{\mu} k_{\nu}
$$

where $\eta_{\mu \nu}$ is metric of auxiliary Minkowski space-time, $x^{\mu}=(t, x . y . z) .{ }^{3}$ Function

$$
h=\frac{m r-e^{2} / 2}{r^{2}+a^{2} \cos ^{2} \theta},
$$

is written in the oblate spheroidal coordinates, and $k_{\mu}$ is a twisting null field which is tangent to the Kerr principal null congruence (PNC) which represents a family of the geodesic null rays - twistors - covering twice the space-time.

Since $k^{\mu}$ is null, it can be represented in the spinor form (1), however, in the Kerr-Schild formalism the projective spinor coordinate $Y$ is used, and the Kerr twistorial congruence is determined by a complex scalar function $Y(x)$, in terms of which the vector $k^{\mu}$ takes the form

$$
k_{\mu} d x^{\mu}=P^{-1}(d u+\bar{Y} d \zeta+Y d \bar{\zeta}-Y \bar{Y} d v),
$$

where $P=2^{-1 / 2}(1+Y \bar{Y})$ is a projective factor and

$$
\begin{aligned}
2^{\frac{1}{2}} \zeta=x+i y, & 2^{\frac{1}{2} \bar{\zeta}}=x-i y \\
2^{\frac{1}{2}} u=z-t, & 2^{\frac{1}{2}} v=z+t
\end{aligned}
$$

are the null Cartesian coordinates.

Therefore, for each point of the Kerr spacetime $x$ we have the pair $\{x, Y(x)\}$ which determines a twistor in the form (4).

The Kerr's twistorial congruence is geodesic and shear-free. Congruences of this type are determined by the Kerr theorem, via the solution $Y(x)$ of

\footnotetext{
${ }^{3}$ We use signature -+++ .
} 


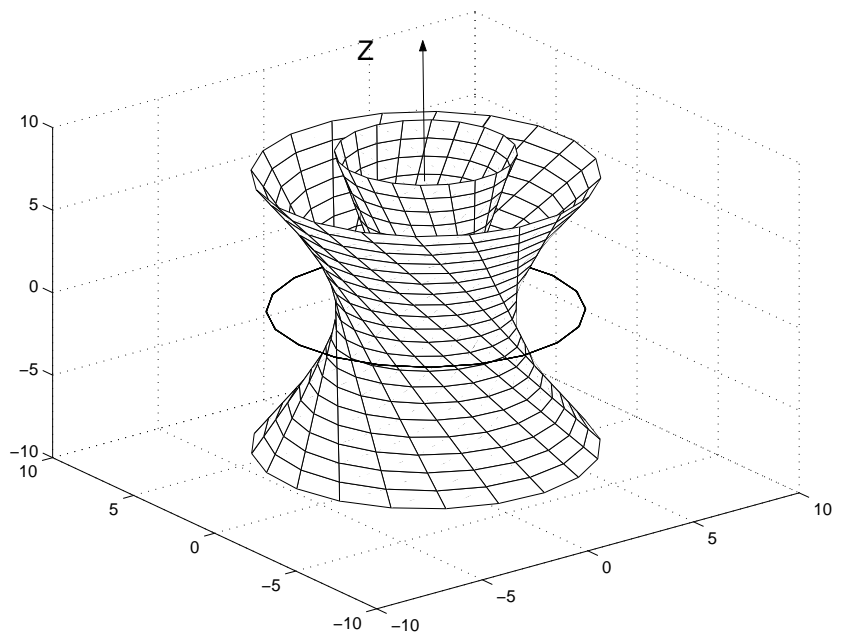

Figure 3: The Kerr singular ring and the Kerr congruence of twistors (PNC). Singular ring is a branch line of space, and PNC propagates from the "negative" sheet of the Kerr space to the "positive" one, covering the space-time twice.

the algebraic equation $F=0$, where $F\left(Y, \lambda_{1}, \lambda_{2}\right)$ is an arbitrary holomorphic function of the projective twistor coordinates ${ }^{4}$

$$
\left\{Y, \quad \lambda_{1}=\zeta-Y v, \quad \lambda_{2}=u+Y \bar{\zeta}\right\}=Z^{a} / Z^{1} .
$$

Function $Y(x)$ allows one to reconstruct from (9) the null vector $k^{\mu}$ which is tangent to the Kerr congruence. This vector is the principal null vector which determine the metric, the Kerr-Newman vector field, and the direction of radiation for radiative Kerr-Schild solutions.

One of the real null tetrad vectors of the Kerr-Schild tetrad is taken proportional to $k^{\mu}, e^{3}=P k^{\mu}$, and tetrad is completed as follows

$$
\begin{aligned}
e^{1} & =d \zeta-Y d v, \quad e^{2}=d \bar{\zeta}-\bar{Y} d v, \\
e^{3} & =d u+\bar{Y} d \zeta+Y d \bar{\zeta}-Y \bar{Y} d v,
\end{aligned}
$$

\footnotetext{
${ }^{4}$ The twistor form (5) is used here. Notice, that the Kerr theorem, which holds one of the prominent positions in twistor theory [11], was practically used by derivation of the Kerr-Schild class of solutions [12], but it was never formulated by Kerr in the twistor terms as well as in the form of a theorem. Meanwhile, the close relation of twistors to the Kerr geometry is not incidental since these ideas were discussed by Roy Kerr and Roger Penrose well before the corresponding publications[23].
} 


$$
e^{4}=d v+h e^{3},
$$

where $e^{1}$ and $e^{2}$ are complex conjugate.

The caustics of the Kerr PNC (singular lines) are determined by the system of equations $F=0, d F / d Y=0$. In the Kerr solution function $F$ is quadratic in $Y$, and these equation can be explicitly resolved [14] leading to the function $Y(x)$ in the form (6) which yields the structure of the Kerr $\mathrm{PNC}$ and the Kerr singular ring shown in Fig. 3.

The twistor $\{x, Y\}$ is a complex null plane at the point $x$ which is spanned by the null vectors $e^{1}$ and $e^{3}$. The real null rays of the Kerr congruence are the real slices of these null planes. The congruence is determined by the real field $k^{\mu}(x)$ (or $e^{3 \mu}=P k^{\mu}$ ) which describes a flow of the lightlike field propagating from the 'negative' sheet of the Kerr space onto 'positive' one through the disk spanned by the Kerr ring. In particular, the Kerr PNC determines a flow of radiation along $e^{3}$, so the outgoing radiation is compensated by an ingoing flow on the negative sheet.

\subsection{Complex Kerr geometry and twistors}

The Kerr twistor-string appears naturally in the Newman-initiated complex representation of the Kerr geometry [25, 22, 14], in which the Kerr solution is generated by a complex source propagating along a complex world line $X_{0}^{\mu}(\tau) \in \mathbf{C M}^{4}$. The Kerr-Schild formalism is well adapted to this representation, since the Kerr-Schild ansatz (7) assumes the auxiliary Minkowski spacetime with Cartesian coordinates $x^{\mu}$, which may be complexified, forming coordinates of $\mathbf{C M}^{4}$. The time parameter $\tau$ is also complex in this representation, $\tau=t+i \sigma$.

The real fields on the real space-time $x^{\mu} \in \mathbf{M}^{4}$ are determined via a complex retarded-time construction, in which the key role is played by the complex light cones, adjoined to the real points of observation $x^{\mu}$ and to the points of emanation on the complex world line $X_{0}^{\mu}(\tau)$.

It is well known that the real light cone may be split on two family of null planes. There are different ways of doing this [22, 14], and all the ways turns out to be close related to twistors. The light cone at a point $x_{0}$ may be written in the spinor form

$$
\mathcal{K}_{x_{0}}=\left\{x: x^{\mu}=x_{0}^{\mu}+\psi^{\alpha} \sigma_{\alpha \dot{\alpha}}^{\mu} \tilde{\psi}^{\alpha}\right\} .
$$


The real null rays of the $\mathcal{K}_{x_{0}}$ correspond to the complex conjugate spinors $\psi^{\alpha}$ and $\tilde{\psi}^{\dot{\alpha}}$. As we discussed in sec.2, varying $\tilde{\psi}$ at fixed $\psi$ one obtains the ('left' ) complex planes, and varying $\psi$ at fixed $\tilde{\psi}$ one obtains another family which we call the right complex planes. We can also use the projective spinor coordinates $Y$ and $\bar{Y}$ and describe the complex null cone as $\mathcal{K}_{x_{0}}=\left\{x_{0}, Y, \bar{Y}\right\}$ and the left (right) null planes of the cone by twistor $\left\{x_{0}, Y\right\}$ (or $\left.\left\{x_{0}, \bar{Y}\right\}\right)$. Notice, that the apex of the light cone $x_{0}$ may also be complex.

To form the retarded-time construction we set the apex of cone $x_{0}$ at a real point $x \in \mathbf{M}^{4}$ and have to find the points of intersection of this light cone with the complex world-line $X_{0}(\tau)$ to determine the value of the retarded-time parameter $\tau_{0}$. In the stationary Kerr case this world-line has the simplest form of the straight timelike line $X_{0}^{\mu}(\tau)=(\tau, 0,0, i a)$. Nevertheless, at this point we meet a peculiarity which complicates the usual retarded-time scheme: there may be four roots for the points of intersection. We can ignore one pair of the roots assigning them to the advanced fields. The residual two roots are linked with the fact that the left and right null planes yield the different points of intersection with a complex world-line. Therefore, we must mark the roots as the left retarded time $\tau_{L}$ and the right one $\tau_{R}$. To get a definiteness we have to use for the roots (for example) only the left null planes, i.e. the holomorphic twistor pairs $\{x, Y\}$. It shows that twistors appear very natural in the complex retarded-time construction.

\section{Complex Kerr string}

In accordance with the above complex representation of the Kerr geometry, the Kerr solution is generated by a complex source propagating along a complex world line $X_{0}^{\mu}(\tau) \in \mathbf{C M}^{4}$. Complex world-line is parametrized by complex time $\tau=t+i \sigma$ containing two parameters $t$ and $\sigma$, and therefore, it describes a world sheet. The complex world line $X_{0}^{\mu}(\tau)$ can be considered as an $N=2$ string in $\mathbf{C M}^{4}[6,22,13]$.

The Lagrangian for this string is $[6,26]^{5}$

$$
\mathcal{L}=\int d \tau d \bar{\tau}\left(\eta_{i \bar{j}}\left(\partial_{\tau} \bar{X}_{0}^{i} \partial_{\bar{\tau}} X_{0}^{j}+\partial_{\bar{\tau}} \bar{X}_{0}^{i} \partial_{\tau} X_{0}^{j}\right)\right.
$$

\footnotetext{
${ }^{5}$ We omit the fermi terms.
} 
As usual, the general solution is given as a sum of the left and right modes,

$$
X_{0}^{i}(t, \sigma)=X_{L}^{i}(\tau)+X_{R}^{i}(\bar{\tau}),
$$

which are not necessarily complex conjugates to each other. The constraints take the form

$$
\eta_{i \bar{j}} \partial_{\tau} \bar{X}_{L}^{i} \partial_{\tau} X_{R}^{j}=0 ; \quad \eta_{i \bar{j}} \partial_{\bar{\tau}} \bar{X}_{L}^{i} \partial_{\bar{\tau}} X_{R}^{j}=0 .
$$

Notice, that the mode expansion contains the hyperbolic basis functions which are not orthogonal over the string length, so similar to the $N=2$ string there are no excitations indeed. Meanwhile, one can see that the straight complex world-line which describes the complex Kerr source,

$$
X_{0} \equiv X_{L}^{\mu}(\tau)=X_{0}(\tau, 0,0, i a),
$$

is a holomorphic left mode which satisfies the equations of motion and constraints.

To obtain the boundary conditions we have to consider an imbedding of this string in $\mathbf{M}^{4}$. It is known that such an imbedding may not be performed retaining the $N=2$ supersymmetry $[6,26] .{ }^{6}$ Therefore, the $N=2$ supersymmetry has to be broken, at least partially [27].

On the other hand, we have to use these left null planes also for the light cones emanating from the points of the complex world line $X_{0}(\tau)$, and note that not all the left null planes reach the real slice.

The null vectors $K^{\mu}=x^{\mu}-X_{0}^{\mu}\left(\tau_{L}\right)$, which belong to the complex light cone, have to connect the complex world-line to the real points $x^{\mu} \in \mathbf{M}^{4}$. This condition gives a restriction on the positions of $\tau_{L}$, which may be easily obtained from the complex retarded-time equation. Let us write the light cone equation in the form

$$
0=K_{\mu} K^{\mu}=\left[\vec{x}-\vec{X}_{0}\left(\tau_{L}\right)\right]^{2}-\left(t-\tau_{L}\right)^{2} .
$$

We assume here that the gauge $X_{0}^{0}\left(\tau_{L}\right)=\tau_{L}=t_{L}+i \sigma_{L}$ is chosen and that the Kerr source is in the rest.

This equation may be split into the retarded-advanced time equations

$$
\left(t-\tau_{L}\right)= \pm \tilde{r},
$$

\footnotetext{
${ }^{6}$ The real spacetimes preserving the $N=2$ supersymmetry have signature $(2,2)$ (Kleinian) or $(4,0)$ (Euclidean).
} 
where $\tilde{r}=\sqrt{\left[\vec{x}-\vec{X}_{0}\left(\tau_{L}\right)\right]^{2}}$ is the complex radial distance which takes the simple form $\tilde{r}=r+i a \cos \theta$ in the Kerr oblate spheroidal coordinate system. Summarizing these relations one obtains from the retarded time equation (18)

$$
t_{L}+i \sigma_{L}=t-r-i a \cos \theta .
$$

The real part of this equation, $t_{L}=t-r$, is similar to the usual retarded-time relation, while the imaginary part,

$$
\sigma_{L}=-a \cos \theta
$$

relates $\sigma_{L}$ with angle $\theta$. Recall, that on the real slice the projective coordinate $Y$ has the form $Y=e^{i \phi} \tan \frac{\theta}{2}$ and fixes the angular directions of twistors. Therefore, the parameter $\sigma_{L}$ fixes $\theta$ direction leaving the coordinate $\phi$ free. One sees that there are the limiting real rays corresponding to polar directions $\theta=0$ and $\theta=\pi$ which correspond to $\sigma_{L}= \pm a$. Only the light cones which are joined to the interval $\sigma_{L} \in \Sigma_{L}=[-a, a]$ have the real slice, and therefore, only a part of the world-sheet, the strip $\sigma \in[-a, a]$, is 'seen' at the real slice and may be imbedded in $\mathbf{M}^{\mathbf{4}}$. It means that the world sheet $(t, \sigma)$ acquires the boundary, forming an open complex string. The end point $\sigma_{L}=-a$ has $\theta=0$, and the corresponding null plane $Y$ has the real slice along the positive semiaxis $z^{+}$, while the end point $\sigma_{L}=+a$ has $\theta=\pi$ and is mapped on the negative semiaxis $z^{-}$. Note, that these semiaxis are exactly the singular semistrings of the real axial stringy system. Therefore the complex string turns out to be a D-string which is stuck to two singular semi-strings of opposite chiralities. Therefore, $z^{ \pm}$singular strings may carry the Chan-Paton factors (chiral currents and traveling waves) which will play the role of quarks with respect to the complex string. ${ }^{7}$

All the intermediate points of the interval $\sigma \in[-a, a]$ have also the joined twistor planes $Y\left(\sigma_{L}\right)$ and the joined real family of semitwistors on the real slice, see Fig. 4.

It should be noted that the $(x, \theta)$ string was discussed in the papers $[7,9,2,28]$ as a prospective alternative for the twistor-string.

\footnotetext{
${ }^{7}$ The relations of the $N=2$ string to the singular cosmic string was discussed for Kleinian spacetimes in[26]. It was mentioned that Kleinian singularities can be blown up, leading to the complete nonsingular metrics, by contrast in the Minkowskian case the stringy singularities are topologically nontrivial.
} 


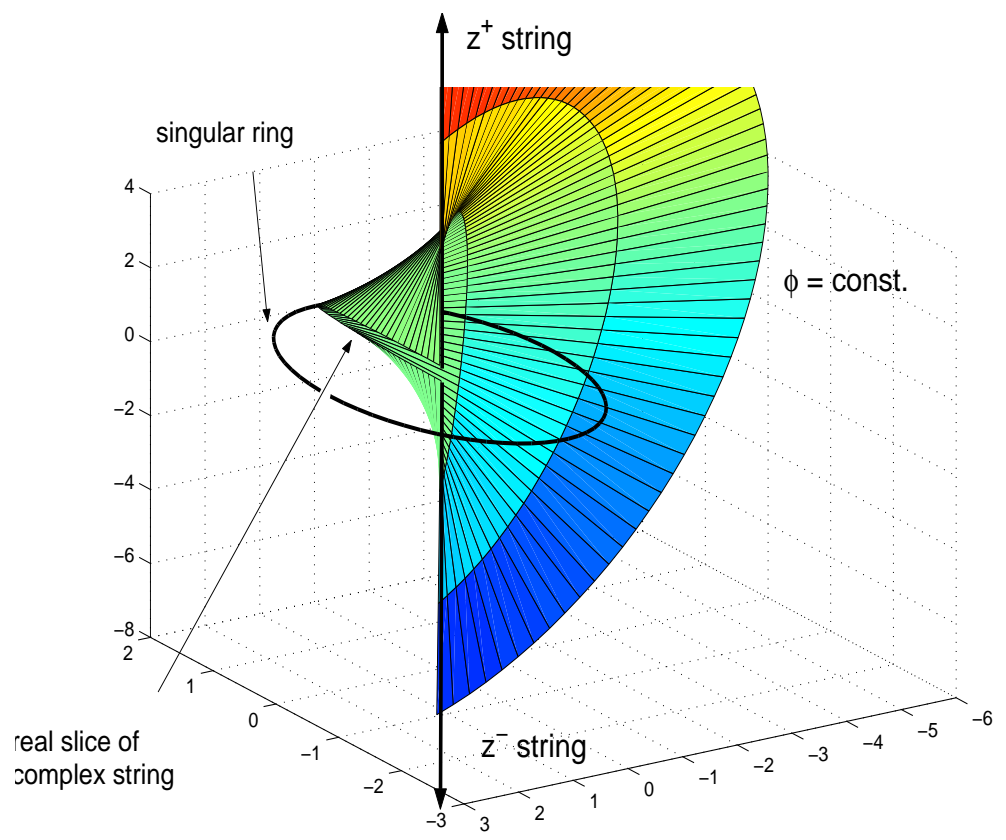

Figure 4: The complex twistor-string is imbedded into the real Kerr geometry. The subset of semi-twistors $(r>0)$ generating the Kerr angular coordinate $\phi=$ const. is shown. The full set of semi-twistors may be obtained by the rotation around z-axis

\subsection{Orientifold}

The considered open twistor-string has, however, a problem with boundary conditions since they may not be defined for the real and imaginary parts simultaneously. This problem is resolved by orientifolding the string, which involves the complex conjugate structure - antiholomorphic complex world line $\bar{X}_{0}^{\mu}(\bar{\tau})$ and corresponding twistors $\left\{\bar{X}_{0}^{\mu}(\bar{\tau}), \bar{Y}\right\}$. This structure is antichiral and carries only the right modes. The chiral and antichiral structures may be joined by orientifolding the complex world-sheet [22].

Similar to the left interval $\sigma_{L} \in \Sigma_{L}=[-a, a]$, the right structure has the right interval $\sigma_{R} \in \Sigma_{R}=[-a, a]$. However, in the right structure worldsheet has an opposite orientation: the end point $\sigma_{R}=-a$ is mapped on the negative semiaxis $z^{-}$, while the end point $\sigma_{R}=+a$ is mapped on the positive semiaxis $z^{+}$. Formation of the orientifold has a few steps. 
The interval $\Sigma_{L}$, parametrized by $0>\theta>\pi$, has to be extended to $0>\theta>2 \pi$. The interval $\Sigma_{R}$ is reversed $\Sigma_{R} \rightarrow \bar{\Sigma}_{R}$ and joined to the interval $\Sigma_{L}$. Therefore, the new interval $\Sigma=\Sigma_{L} \cup \bar{\Sigma}_{R}$ covers twice the initiate one going the second time in opposite direction and forming a closed loop.

The string modes are extended to $\pi>\theta>2 \pi$ in the standard manner [29] which allows one to consider the open string as a closed one:

$$
X_{L}(\theta+\pi)=X_{R}(\theta), \quad X_{R}(\theta+\pi)=X_{L}(\theta)
$$

This operation is accompanied by the transformations of the null planes $\bar{Y} \rightarrow-1 / Y$ (right $\rightarrow$ left $)$ and by the parity $r \rightarrow-r$.

As a result the string acquires the right modes and turns into a closed, but folded one $[22,13,5]$. The final step is the setting of the $\mathbf{Z}_{\mathbf{2}}$ equivalence of the left and right structures.

It should be noted that target space of this string turns out to be equivalent to the 'diagonal' of the $\mathbf{C P}^{\mathbf{3}} \times \mathbf{C P}^{* \mathbf{3}}$ which was discussed by Witten in the end of paper [1] and also long ago [30] as a formulation of the classical Yang-Mills theory. The used in the Kerr spinning particle equivalent representation $\left\{X_{0}, Y\right\}$ may be preferable in some cases [28], since the spacetime coordinates appear explicitly in this form and geometrical interpretation looks more transparent.

The extra complex coordinate $Y$ is free for the complex $N=2$ string, but it acquires the $\sigma$ dependence by imbedding to the real slice. In the $\mathbf{C P}^{3}$ representation of twistor-string it plays the role of a target coordinate. However, as it was discussed in [6], there are some evidences that the worldsheet of the $N=2$ string is dual to its target space, and is indeed fourdimensional. It suggests that $Y$ may play a double role: of the world-sheet and target coordinate, which turns the $N=2$ string into a membrane as it was discussed in [6, 31]. Similar, in it was suggested in the work [9] that coordinate $Y$ must not acquire the stringy excitations, and being to considered in the frame of the string field theory, acts as a carrier of the field excitations. These conjectures are supported by the Kerr spinning particle.

\section{Conclusion}

A few related important problems have been left without treatment in this paper. Among them are the supergeneralization of the model and twist of 
the spin-structure.

A scheme for supergeneralization of the Kerr solution was considered in [27], and it was shown that the $N=2$ supersymmetry turns out to be broken in $\mathbf{M}^{4}$, which yields a residual nonlinear realization of supersymmetry in the frame of the broken $N=2$ supergravity. Therefore, the treatment of the supersymmetry in this model turns out to be more complicated that in the Witten-Ferber approach $[1,30]$.

The twist of spin-structure $[1,2,32]$ has to be incorporated in the model of the Kerr spinning particle to provide a natural description of fermions in terms of the half-integer excitations. In the Kerr geometry twist is connected with topological structure of the axial singular strings $[33]^{8}$, which turns out to be related to the deep questions of the topological field theory $[32,33,34]$. On the other hand, twist is close related to supersymmetry. Preliminary treatment shows that the Kerr-Schild formalism is perfectly matched to twist, however, some basic relations have to be recalculated, which demands a special treatment.

Both these problems we intend to discuss elsewhere.

Author is thankful to Organizing Committee of the Spin04-Praha Conference for invitation and financial support, and also to the participants of the Conference SPIN04-Praha and the Trieste Symposium SPIN2004: A. Efremov, O. Teryaev, A. Dorokhov and O. Selugin for interest in this work and very useful conversations. Author would also like to thank B. Dubrovin and A. Dabholkar for the useful discussions at Trieste.

\section{References}

[1] E. Witten, Perturbative Gauge Theory as a String Theory in Twistor Space, Comm. Math. Phys. 252, 189 (2004), hep-th/0312171.

[2] V.P. Nair, A Current Algebra for Some Gauge Theory Amplitudes, Phys. Lett. B214, 215 (1988).

\footnotetext{
${ }^{8}$ Note, that the axial singularities of the Kerr spinning particle are different from the black hole singularities considered in [33]
} 
[3] G. Chalmers and W. Siegel, Simplifying Algebra in Feynman Graphs. Part III, Phys.Rev. D 63, 125027 (2001), hep-th/0101025, Part II, Phys.Rev. D 59, 045013 (1999), hep-ph/9801220; Part I, Phys.Rev. D 59, 045012 (1999), hep-ph/9708251.

[4] Z. Bern, L.Dixon and D.Kosower, Progress in One-loop QCD Computations. Ann.Rev.Nucl.Part.Sci. 46, 109(1996).

[5] A. Burinskii, Twistorial Analiticity and Three Stringy Structures of the Kerr Spinning Particle, Phys.Rev. D 70 (2004) 086006, hep-th/0406063.

[6] H. Ooguri, C. Vafa, Geometry of $N=2$ string, Nucl. Phys. B 361, 469(1991); Selfduality and $N=2$ String Magic, Mod. Phys. Lett A5, 1389 (1990).

[7] N. Berkovits, Self-Dual Super-Yang-Mills as a String Theory in $(x, \theta)$ Space, JHEP 0405 (2004) 034, hep-th/0403280

[8] W. Siegel, The $N=2(4)$ String is Selfdual $N=4$ Yang-Mills, Phys.Rev. D 46 (1992) R3235, hep-th/9205075

[9] O. Lechtenfeld and A. Popov, Supertwistors and Cubic String Field Theory for Open $N=2$ Strings, Phys. Lett. B 598 (2004) 113, hepth/0406179.

[10] Y. Abe, V.P. Nair and M. Park, Multigluon amplitudes, $\mathcal{N}=4$ constraints and the WZW model, hep-th/0408191.

[11] R. Penrose, Twistor Algebra, J. Math. Phys. 8 (1967) 345.

[12] G.C. Debney, R.P. Kerr, A.Schild, Solutions of the Einstein and Einstein - Maxwell Equations, J. Math. Phys. 10, 1842(1969).

[13] A. Burinskii, Orientifold D-string in the source of the Kerr spinning particle, Phys.Rev. D 68, 105004 (2003).

[14] A. Burinskii, Complex Kerr geometry and nonstationary Kerr solutions, Phys. Rev. D 67, 124024 (2003); Complex structure of Kerr geometry and rotating "photon rocket" solutions, Clas. Quant. Gravity 20, 905 (2003). 
[15] A. Burinskii, Axial Stringy System of the Kerr Spining Particle, Grav.\&Cosmology, 10, n.1-2 (37-38), 50 (2004), hep-th/0403212.

[16] A.Ya. Burinskii, Microgeons with spin, Sov. Phys. JETP, 39, 193(1974).

[17] D. Ivanenko and A.Ya. Burinskii, Gravitational Strings in the Models of Elementary Particles, Izvestiya Vuzov Fiz. 5, 135 (1975) (in russian).

[18] A. Dabholkar, G. Gibbons, J.A. Harvey, F. Ruiz Ruiz, Superstrings and solitons, Nucl. Phys. B 340, 33 (1990)

[19] A.Sen, Macroscopic Charged Heterotic String, Nucl. Phys. B388, 457 (1992), hep-th/9206016,

[20] E. Witten, Superconducting Strings, Nucl.Phys., B249, 557(1985).

[21] A.Y. Burinskii, Some Properties of the Kerr Solution to Low Energy String Theory, Phys. Rev. D 52, 5826 (1995).

[22] A.Ya. Burinskii, The Kerr geometry, complex world lines and hyperbolic strings, Phys.Lett. A 185, 441 (1994); String-like Structures in Complex Kerr Geometry. In: Relativity Today, edited by R.P.Kerr and Z.Perjés (Akadémiai Kiadó, Budapest, 1994), p.149; gr-qc/9303003, hepth/9503094.

[23] R.P. Kerr, privat communication, see also the Acknowledgment of Ref. [11].

[24] A. Burinskii, Supersymmetric superconducting bag as a source of the Kerr spinning particle, Grav.\& Cosmology, 8, 261 (2002).

[25] E.T. Newman, Maxwell equations and complex Minkowski space, J. Math. Phys. 15, 44 (1974); On a Classical, Geometric Origin of Magnetic Moments, Spin-Angular Momentum and the Dirac Gyromagnetic Ratio, Phys.Rev. D 65, 104005 (2002).

[26] J. Barrett, G.W. Gibbons, M.J. Perry, C.N. Pope and P. Ruback, Kleinian Geometry and the $N=2$ Superstring, Int. J. Mod. Phys. A 9(1994) 1457. 
[27] A. Burinskii, Kerr Spinning Particle, Strings and Superparticle Models. Class.Quant.Grav. 16, 3497 (1999), Phys.Rev. D 57, 2392 (1998).

[28] W. Siegel, Untwisting the twistor superstring, hep-th/0404255.

[29] M.B. Green, J. Schwarz, and E. Witten, Superstring Theory, v.I, Cambridge Univ. Press, 1987.

[30] E. Witten, An Interpretation of Classical Yang-Mills Theory, Phys. Lett. B 77, 394 (1978),

J. Isenberg, P. B. Yasskin and P.S. Green, Nonselfdual Gauge Fields, Phys. Lett. B 78, 464 (1978),

[31] S. V. Ketov, From $N=2$ Strings to $F \& 3$ Theory, Nucl.Phys.Proc.Suppl. 52 A (1997) 335-338, hep-th/9606142.

[32] N. Berkovits and Vafa, $N=4$ Topological Strings, Nucl. Phys. B433 (1995) 123.

[33] T. Eguchi, Topological Field Theories and the Space-Time Singularity, Mod.Phys.Lett. A7 , 85 (1992), hep-th/9111001.

[34] E. Witten, Topological Sigma Models, Comm. Math. Phys. 118 (1988) 411; Topological Quantum Field Theory, Comm. Math. Phys. 117 (1988) 353. 